\title{
Minimax Estimation of the Parameter of Exponential Distribution based on Record Values
}

\author{
Lanping Li \\ Department of Basic Subjects, Hunan University of Finance and Economics, Changsha 410205, P.R.China \\ E-mail: lilanping1981@163.com
}

\begin{abstract}
Bayes estimators of the parameter of exponential distribution are obtained with noninformative quasi-prior distribution based on record values under three loss functions. These functions are weighted squared error loss, square log error loss and entropy loss functions. Finally the minimax estimators of the parameter are obtained by using Lehmann's theorem. Comparisons in terms of risks with the estimators of parameter under three loss functions are also studied.
\end{abstract}

Index Terms-Bayes Estimator, Minimax Estimator, Squared Log Error Loss, Entropy Loss, Record Value

\section{Introduction}

Record values and the associated statistics area of interest and important in many real life applications involving data relating to meteorology, sport, economics and life testing.

Set

$$
Y_{n}=\max \left\{X_{1}, X_{2}, \cdots, X_{n}\right\}, n \geq 1 \text {, }
$$

we say that $X_{j}$ is an upper record and denoted by $X_{U(j)}$ if $Y_{j}>Y_{j-1}, j>1$.

The detail about record values can refer Arnold et al.(1998), Raqab (2002), Jaheen (2004) and Ahmadi et al.(2005) and references therein.

Exponential distribution is one of the most commonly used models in life-testing and reliability studies. Inferential issues concerning the exponential distribution, and applications in the context of lifetesting and reliability, have been extensively discussed by many scholars. A great deal of research has been done on estimating the parameters of the exponential distribution using both classical and Bayesian techniques. See for example Bain (1978), Chandrasekar et al. (2002), Jaheen (2004), and Ahmadi et al. (2005) and references therein.

This paper is devoted to the minimax estimation problem of the unknown scale parameter $\theta$ in the exponential distribution with probability density function (pdf)

$$
f(x ; \theta)=\theta \exp (-\theta \mathrm{x}), \quad x>0, \theta>0
$$

and cumulative distribution function(cdf)

$$
F(x ; \theta)=1-\exp (-\theta \mathrm{x}), \quad x>0, \theta>0
$$

where $f(x ; \theta)$ denotes the conditional pdf of random variable(r.v.)X given $\theta$.

This paper will discuss the minimax estimation of the parameter of exponential distribution based on record values.

\section{Preliminaries}

\subsection{Maximum Likelihood Estimation}

Let $X_{1}, X_{2}, \cdots$ be a sequence of independent and identically distributed (iid) random variables with cdf $F(x ; \theta)$ and pdf $f(x ; \theta)$.

In the following discussion, we always suppose that we observe $n$ upper record values

$$
X_{U(1)}=x_{1}, X_{U(2)}=x_{2}, \cdots, X_{U(n)}=x_{n}
$$

drawn from the exponential model with pdf given by (1)

The joint distribution of $X_{U(1)}, X_{U(2)}, \cdots, X_{U(n)}$ is given (see Arnold et al. (1998)) by

$$
\begin{aligned}
& f_{1,2, \cdots, n}(x ; \theta)=f\left(x_{n} ; \theta\right) \prod_{i=1}^{n-1} h\left(x_{i} ; \theta\right), \\
& 0<x_{1}<\cdots<x_{n}
\end{aligned}
$$

Where $x=\left(x_{1}, x_{2}, \cdots, x_{n}\right), h\left(x_{i} ; \theta\right)=\frac{f\left(x_{i} ; \theta\right)}{1-F\left(x_{i} ; \theta\right)}$

Since the marginal pdf of $X_{U(n)}$ is given (see Arnold et al. (1998)) by 


$$
\begin{aligned}
f_{n}\left(x_{n} ; \theta\right) & =f\left(x_{n} ; \theta\right) \frac{\left[-\ln \left(1-F\left(x_{n} ; \theta\right)\right]^{n-1}\right.}{(n-1) !} \\
& =\frac{1}{\Gamma(n)} \theta^{n} x_{n}^{n-1} e^{-\theta x_{n}}, x>0
\end{aligned}
$$

Thus

$$
X_{U(n)} \sim \Gamma(n, \theta)
$$

and

$$
E X_{U(n)}=n / \theta
$$

The likelihood function based $\mathrm{n}$ upper record values is given by

$$
l(\theta \mid x)=f_{1,2, \cdots, n}(x ; \theta)=\theta^{n} \exp \left(-\theta x_{n}\right)
$$

and the log-likelihood function may be written as

$$
L(\theta \mid x)=\ln l(\theta \mid x)=n \ln \theta-\theta x_{n}
$$

Upon differentiating (6) with respect to $\theta$ and equating each results to zero, the MLE of $\theta$ is given by

$$
\frac{d \ln L(\theta \mid x)}{d \theta}=\frac{n}{\theta}-x_{n}=0
$$

Then, the MLE of $\theta$ is

$$
\hat{\delta}_{M L E}=\frac{n}{X_{U(n)}}
$$

\subsection{Loss Function}

In statistical decision theory and Bayesian analysis, loss function plays an important role in it and the most common loss are symmetric loss function ,especially squared error loss function are considered most. Under squared loss function, it is to be thought the overestimation and underestimation have the same estimated risks. However, in many practical practical problems, overestimation and underestimation will have different consequences. To overcome this difficulty, Varian(1975)and Zellner(1986)proposed an asymmetric loss function known as the LINEX loss function, Podder et al.(2004) proposed a new asymmetric loss function for scale parameter estimation. See also Kiapoura and Nematollahib(2011), Mahmoodi, and Farsipour(2006). This loss function is called squared $\log$ error $\operatorname{loss}(\mathrm{SLE})$ is

$$
L(\theta, \delta)=(\ln \delta-\ln \theta)^{2}
$$

Which is balanced and $L(\theta, \delta) \rightarrow \infty$ as $\delta \rightarrow 0$ or $\infty$. This loss is not always convex, it is convex for $\frac{\delta}{\theta} \leq e$ and concave otherwise, but its risk function has minimum w.r.t.

$$
\hat{\delta}_{S L}=\exp [E(\ln \theta \mid X)] .
$$

In many practical situations, it appears to be more realistic to express the loss in terms of the ratio $\hat{\theta} / \theta$. In this case, Dey et al. (1987) pointed out that a useful asymmetric loss function is entropy loss function:

$$
L(\hat{\theta}, \theta)=\frac{\delta}{\theta}-\ln \frac{\delta}{\theta}-1
$$

Whose minimum occurs at $\delta=\theta$. Also, this loss function has been used in Singh et al. (2011), Li and Ren(2012) .The Bayes estimator under the entropy loss is denoted by $\hat{\theta}_{B E}$, given by

$$
\hat{\delta}_{B E}=\left[E\left(\theta^{-1} \mid X\right)\right]^{-1} .
$$

\section{Bayes Estimation}

In this section, we estimate $\theta$ by considering weighted square error loss, squared log error loss and entropy loss functions.

We further assume that some prior knowledge about the parameter $\theta$ is available to the investigation from past experience with the exponential model. The prior knowledge can often be summarized in terms of the socalled prior densities on parameter space of $\theta$. In the following discussion, we assume the following Jeffrey's non-informative quasi-prior density defined as,

$$
\pi(\theta) \propto \frac{1}{\theta^{d}}, \quad \theta>0
$$

Hence, $d=0$ leads to a diffuse prior and $d=1$ to a non-informative prior.

Combing the likelihood function (5) and the prior density(10), we obtain the posterior density of $\theta$ is

$$
h(\theta \mid x)=\frac{x_{n}^{n-d+1}}{\Gamma(n-d+1)} \theta^{(n-d+1)-1} e^{-\theta x_{n}}
$$

This is a Gamma distribution. $\Gamma\left(n-d+1, x_{n}\right)$, where $x_{n}$ is the observation of $X_{U(n)}$. 
Theorem3.1. Under the weighted square error loss function

$$
L_{1}(\theta, \delta)=\frac{(\delta-\theta)^{2}}{\theta^{2}}
$$

Then (i) the Bayes estimator under the weighted square error loss function is given by

$$
\hat{\delta}_{B S}=\frac{E\left[\theta^{-1} \mid X\right]}{E\left[\theta^{-2} \mid X\right]}=\frac{n-d-1}{X_{U(n)}}
$$

(ii) the Bayes estimator under the squared log error loss function is come out to be

$$
\hat{\delta}_{S L}=\exp [E(\ln \theta \mid X)]=\frac{e^{\Psi(n)}}{X_{U(n)}}
$$

(iii) the Bayes estimator under the entropy loss function is obtained as

$$
\hat{\delta}_{B E}=\left[E\left(\theta^{-1} \mid X\right)\right]^{-1}=\frac{n-d}{X_{U(n)}}
$$

Proof. (i) by formula (11),we know that

$$
\theta \mid X \square \Gamma\left(n-d+1, X_{U(n)}\right),
$$

Then

$$
\begin{aligned}
& E\left[\theta^{-1} \mid X\right]=\frac{X_{U(n)}}{n-d}, \\
& E\left[\theta^{-2} \mid X\right]=\frac{X_{U(n)}^{2}}{(n-d)(n-d-1)}
\end{aligned}
$$

Thus, the Bayes estimator under the weighted square error loss function is given by

$$
\begin{aligned}
\hat{\delta}_{B S} & =\frac{E\left[\theta^{-1} \mid X\right]}{E\left[\theta^{-2} \mid X\right]} \\
& =\frac{X_{U(n)} /(n-d)}{X_{U(n)}^{2} /(n-d)(n-d-1)}=\frac{n-d-1}{X_{U(n)}}
\end{aligned}
$$

(ii) Using (12),

$$
\begin{aligned}
& E(\ln \theta \mid X) \\
& =\frac{X_{U(n)}^{n-d+1}}{\Gamma(n-d+1)} \int_{0}^{\infty} \ln \theta \cdot \theta^{(n-d+1)-1} e^{-\theta X_{U(n)}} d \theta \\
& =\frac{d}{d n} \ln \Gamma(n-d+1)-\ln X_{U(n)} \\
& =\Psi(n-d+1)-\ln X_{U(n)}
\end{aligned}
$$

Where

$$
\Psi(n)=\frac{d}{d n} \ln \Gamma(n)=\int_{0}^{+\infty} \frac{\ln y \cdot y^{n-1} e^{-y}}{\Gamma(n)} d y
$$

is a Digamma function.

Then the Bayes estimator under the squared log error loss function is come out to be

$$
\hat{\delta}_{S L}=\exp [E(\ln \theta \mid X)]=\frac{e^{\Psi(n-d+1)}}{X_{U(n)}}
$$

(iii) By Eqs.(10) and (17), the Bayes estimator under the entropy loss function is given by

$$
\hat{\delta}_{B E}=\left[E\left(\theta^{-1} \mid X\right)\right]^{-1}=\frac{n-d}{X_{U(n)}} .
$$

\section{Minimax Estimation}

The most important elements in the minimax approach are the specification of the prior distribution and the loss functions by using a Bayesian method.

The derivation of minimax estimators depends primarily on a theorem due to Lehmann which can be stated as follows:

Lemma4.1(Lehmann's Theorem) If $\tau=\left\{F_{\theta} ; \theta \in \Theta\right\}$ be a family of distribution functions and $D$ a class of estimators of $\theta$.Suppose that $\delta^{\bullet} \in D$ is a Bayes estimator against a prior distribution $\delta^{\bullet}(\theta)$ on $\Theta$, and $R\left(\delta^{\bullet}, \theta\right)$ equals constant on $\Theta$; then $\delta^{\bullet}$ is a minimax estimator of $\theta$.

Theorem 4.1 Let $X_{1}, X_{2}, \ldots, X_{n}$ be a random sample drawn from the density (1),

then $\hat{\delta}_{B S}=\frac{n-d-1}{X_{U(n)}}$ is the minimax estimator of 
the parameter $\theta$ for the weighted square error loss function of the type (13).

Theorem 4.2 Let $X_{1}, X_{2}, \ldots, X_{n}$ be a random sample drawn from the density (1),

$$
\text { then } \hat{\delta}_{S L}=\frac{e^{\Psi(n-d+1)}}{X_{U(n)}} \text { is the minimax estimator of }
$$

the parameter $\theta$ for the squared log error loss function of the type (8).

Theorem 4.3 Let $X_{1}, X_{2}, \ldots, X_{n}$ be a random sample drawn from the density (1),

then $\hat{\delta}_{B E}=\frac{n-d}{X_{U(n)}}$ is the minimax estimator of the parameter $\theta$ for the entropy loss function of the type (9).

Proof. First we have to prove the theorem 4.1. To prove the theorem we shall use Lehmann's theorem, which has been stated before.

For this, first we have to find the Bayes estimator $\delta$ of $\theta$. Then if we can show that the risk of $d$ is constant, then the theorem3.1 will be proved.

The risk function of the estimator $\hat{\delta}_{B S}=\frac{n-d-1}{X_{U(n)}}$ is

$$
\begin{aligned}
R(\theta)=E\left[L\left(\theta, \frac{n-d-1}{X_{U(n)}}\right)\right] \\
=E\left[\left(\frac{(n-d-1) / X_{U(n)}-\theta}{\theta}\right)^{2}\right] \\
=\frac{1}{\theta^{2}}\left[(n-d-1)^{2} E\left(X_{U(n)}^{-2}\right)\right. \\
\left.\quad-2 \theta(n-d-1) E\left(X_{U(n)}^{-1}\right)+\theta^{2}\right]
\end{aligned}
$$

From the conclusion $X_{U(n)} \sim \Gamma(n, \theta)$, we have

$$
\begin{aligned}
& E\left(X_{U(n)}^{-1}\right)=\frac{\theta}{n-1}, \\
& E\left(X_{U(n)}^{-2}\right)=\frac{\theta^{2}}{(n-1)(n-2)}
\end{aligned}
$$

Then

$$
\begin{gathered}
R(\theta)=\frac{1}{\theta^{2}}\left[(n-d-1)^{2} \frac{\theta^{2}}{(n-d)(n-2)}\right. \\
\left.-2 \theta(n-d-1) \frac{\theta}{n-d}+\theta^{2}\right] \\
=1-2 \frac{n-d-1}{n-d}+\frac{(n-d-1)^{2}}{(n-d)(n-2)}
\end{gathered}
$$

Then $R(\theta)$ is a constant .So, according to the Lehmann's theorem it follows that, $\hat{\delta}_{B S}=\frac{n-d-1}{X_{U(n)}}$ is the minimax estimator for the parameter $\theta$ of the exponential distribution under the weighted square error loss function of the form (12).

Now we are going to prove the theorem4.2. The risk function of the estimator $\hat{\delta}_{S L}=\frac{e^{\Psi(n-d+1)}}{X_{U(n)}}$ is

$$
\begin{gathered}
R(\theta)=E\left[L\left(\theta, \hat{\delta}_{S L}\right)\right]=E\left[\left(\ln \hat{\delta}_{S L}-\ln \theta\right)^{2}\right] \\
=E\left[\ln \hat{\delta}_{S L}\right]^{2}-2 \ln \theta \cdot E \ln \left[\hat{\delta}_{S L}\right]+(\ln \theta)^{2}
\end{gathered}
$$

From the conclusion $X_{U(n)} \sim \Gamma(n, \theta)$, we have

$$
E\left(\ln X_{U(n)}\right)=\Psi(n)-\ln \theta
$$

Thus

$$
\begin{aligned}
& E\left[\ln \hat{\delta}_{S L}\right]=E\left(\Psi(n-d+1)-\ln X_{U(n)}\right) \\
= & \Psi(n-d+1)-(\Psi(n)-\ln \theta) \\
= & \Psi(n-d+1)-\Psi(n)+\ln \theta \\
E & {\left[\ln \hat{\delta}_{S L}\right]^{2}=E\left(\Psi(n-d+1)-\ln X_{U(n)}\right)^{2} } \\
= & \Psi^{2}(n-d+1)-2 \Psi(n-d+1) E\left(\ln X_{U(n)}\right) \\
& +E\left[\left(\ln X_{U(n)}\right)^{2}\right]
\end{aligned}
$$

Using the fact

$$
\begin{aligned}
\Psi^{\prime}(n)= & \int_{0}^{\infty} \frac{(\ln y)^{2} y^{n-1} e^{-y}}{\Gamma(n)} d y \\
& \quad-\int_{0}^{\infty} \frac{(\ln y)^{2} y^{n-1} e^{-y}}{\Gamma(n)} \Psi(n) d y, \\
= & E\left[(\ln Y)^{2}\right]-\Psi^{2}(n)
\end{aligned}
$$


where $Y \sim \Gamma(n, 1)$.and we can show that

$$
Y=X_{U(n)} \theta \sim \Gamma(n, 1)
$$

Thus

$$
\begin{aligned}
& \Psi^{2}(n)+\Psi^{\prime}(n) \\
& =E\left[(\ln Y)^{2}\right]=E\left[\left(\ln X_{U(n)}+\ln \theta\right)^{2}\right] \\
& =E\left[\left(\ln X_{U(n)}\right)^{2}\right]+2 \ln \theta \cdot E\left(\ln X_{U(n)}\right)+(\ln \theta)^{2} \\
& =E\left[\left(\ln X_{U(n)}\right)^{2}\right]+2 \ln \theta \cdot(\Psi(n)-\ln \theta)+(\ln \theta)^{2}
\end{aligned}
$$

Then we get the fact

$$
\begin{aligned}
E\left[\left(\ln X_{U(n)}\right)^{2}\right]= & \Psi^{2}(n)+\Psi^{\prime}(n) \\
& -2 \ln \theta \cdot \Psi(n)+(\ln \theta)^{2}
\end{aligned}
$$

Therefore

$$
\begin{aligned}
E & {\left[\ln \hat{\delta}_{S L}\right]^{2} } \\
= & \Psi^{2}(n-d+1)-2 \Psi(n-d+1) E\left(\ln X_{U(n)}\right) \\
& +E\left[\left(\ln X_{U(n)}\right)^{2}\right] \\
= & \Psi^{2}(n-d+1)-2 \Psi(n-d+1)[\Psi(n)-\ln \theta] \\
& \quad+\Psi^{2}(n)+\Psi^{\prime}(n)-2 \ln \theta \cdot \Psi(n)+(\ln \theta)^{2}
\end{aligned}
$$

$$
R(\theta)
$$

$$
\begin{aligned}
= & E\left[\ln \hat{\delta}_{S L}\right]^{2}-2 \ln \theta \cdot E \ln \left[\hat{\delta}_{S L}\right]+(\ln \theta)^{2} \\
= & \Psi^{2}(n-d+1)-2 \Psi(n-d+1)[\Psi(n)-\ln \theta] \\
& +\Psi^{2}(n)+\Psi^{\prime}(n)-2 \ln \theta \cdot \Psi(n)+(\ln \theta)^{2} \\
& -2 \ln \theta \cdot[\Psi(n-d+1)-\Psi(n)+\ln \theta]+(\ln \theta)^{2} \\
= & \Psi^{2}(n-d+1) \\
& -2 \Psi(n) \Psi(n-d+1)+\Psi^{2}(n)+\Psi^{\prime}(n)
\end{aligned}
$$

Then $R(\theta)$ is a constant. So, according to the Lehmann's theorem it follows that, $\hat{\delta}_{S L}=\frac{e^{\Psi(n-d+1)}}{X_{U(n)}}$ is the minimax estimator for the parameter $\theta$ of the exponential distribution under the squared log error loss function of the form (8).

Finally we are going to prove the theorem4.3.The risk function of the estimator $\hat{\delta}_{B E}=\frac{n-d}{X_{U(n)}}$ is

$$
\begin{aligned}
R(\theta)=E[L(\theta,)] & =E\left[\frac{n-d}{\theta X_{U(n)}}-\ln \frac{n-d}{\theta X_{U(n)}}+1\right] \\
= & \frac{n-d}{\theta} E\left[\frac{1}{X_{U(n)}}\right] \\
& -\ln (n-d)+\ln \theta+E\left[\ln X_{U(n)}\right]+1 \\
= & \frac{n-d}{n-1}-\ln (n-d)+\ln \theta+\Psi(n)-\ln \theta+1 \\
= & \frac{n-d}{n-1}-\ln (n-d)+\Psi(n)+1
\end{aligned}
$$

Then $R(\theta)$ is a constant .So, according to the Lehmann's theorem it follows that, $\hat{\delta}_{B E}=\frac{n-d}{X_{U(n)}}$ is the minimax estimator for the parameter $\theta$ of theexponential distribution under the entropy loss function of the form (9).

\section{Risk Function}

The risk functions of the estimators $\hat{\theta}_{B S}, \hat{\theta}_{B L}$ and $\hat{\theta}_{B E}$ relative to squared error loss

$$
L(\hat{\theta}, \theta)=(\delta-\theta)^{2}
$$

are denoted by $R\left(\hat{\theta}_{B S}\right), R\left(\hat{\theta}_{B L}\right)$ and $R\left(\hat{\theta}_{B E}\right)$, respectively, are can be easily shown as

$$
\begin{aligned}
& R\left(\hat{\delta}_{B S}\right)=E\left[L\left(\hat{\delta}_{B S}, \theta\right)\right] \\
& =E\left[\left(\hat{\delta}_{B S}-\theta\right)^{2}\right]=E\left[\left(\frac{n-d-1}{X_{U(n)}}-\theta\right)^{2}\right] \\
& =\int_{0}^{\infty}\left[\frac{(n-d-1)^{2}}{x^{2}}-\frac{2(n-d-1) \theta}{x}+\theta^{2}\right] f_{X_{U(n)}}(x) d x \\
& =\int_{0}^{\infty}\left[\frac{(n-d-1)^{2}}{x^{2}}-\frac{2(n-d-1) \theta}{x}+\theta^{2}\right] \frac{\theta^{n}}{\Gamma(n)} x^{n-1} e^{-\theta x} d x, \\
& =\theta^{2}\left[\frac{(n-d-1)^{2}}{(n-1)(n-2)}-\frac{2(n-d-1)}{n-1}+1\right]
\end{aligned}
$$




$$
\begin{aligned}
& R\left(\hat{\delta}_{S L}\right)=E\left[\left(\hat{\delta}_{S L}-\theta\right)^{2}\right]=E\left[\left(\frac{e^{\Psi(n-d+1)}}{X_{U(n)}}-\theta\right)^{2}\right] \\
& =\int_{0}^{\infty}\left[\frac{e^{2 \Psi(n-d+1)}}{x^{2}}-\frac{2 e^{\Psi(n-d+1)} \theta}{x}+\theta^{2}\right] f_{X_{U(n)}}(x) d x \\
& =\int_{0}^{\infty}\left[\frac{e^{2 \Psi(n-d+1)}}{x^{2}}-\frac{2 e^{\Psi(n-d+1)} \theta}{x}+\theta^{2}\right] \frac{\theta^{n}}{\Gamma(n)} x^{n-1} e^{-\theta x} d x, \\
& =\theta^{2}\left[\frac{e^{2 \Psi(n-d+1)}}{(n-1)(n-2)}-\frac{2 e^{\Psi(n-d+1)}}{n-1}+1\right]
\end{aligned}
$$

$$
\begin{aligned}
& R\left(\hat{\delta}_{B E}\right)=E\left[L\left(\hat{\delta}_{B E}, \theta\right)\right] \\
& =E\left[\left(\hat{\delta}_{B E}-\theta\right)^{2}\right]=E\left[\left(\frac{n-d}{X_{U(n)}}-\theta\right)^{2}\right] \\
& =\int_{0}^{\infty}\left[\frac{(n-d)^{2}}{x^{2}}-\frac{2(n-d) \theta}{x}+\theta^{2}\right] f_{X_{U(n)}}(x) d x \\
& =\int_{0}^{\infty}\left[\frac{(n-d)^{2}}{x^{2}}-\frac{2(n-d) \theta}{x}+\theta^{2}\right] \frac{\theta^{n}}{\Gamma(n)} x^{n-1} e^{-\theta x} d x \\
& =\theta^{2}\left[\frac{(n-d)^{2}}{(n-1)(n-2)}-\frac{2(n-d)}{n-1}+1\right]
\end{aligned}
$$

In the Fig.1-4, we have plotted the ratio of the risk functions to $\theta^{2}$,i.e.

$$
\frac{R\left(\hat{\delta}_{B S}\right)}{\theta^{2}}=B_{1}, \quad \frac{R\left(\hat{\delta}_{S L}\right)}{\theta^{2}}=B_{2}, \text { and } \frac{R\left(\hat{\delta}_{B E}\right)}{\theta^{2}}=B_{3}
$$

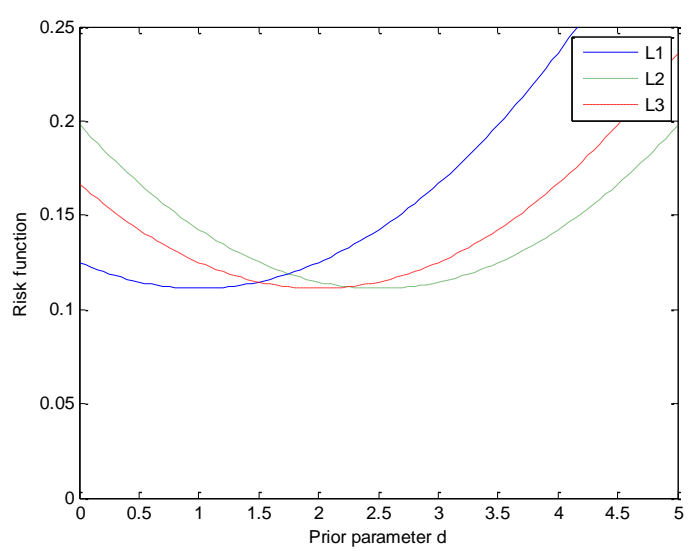

Fig. 1: Ratio of the risk functions with $\mathrm{n}=10$

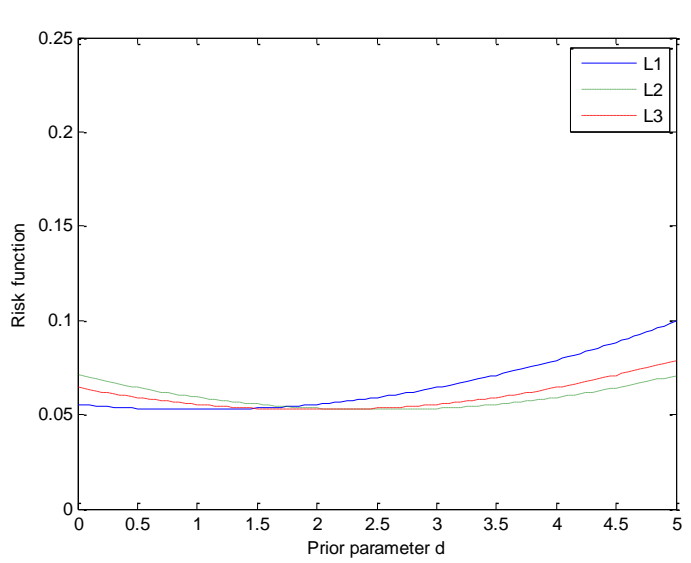

Fig. 2: Ratio of the risk functions with $n=20$

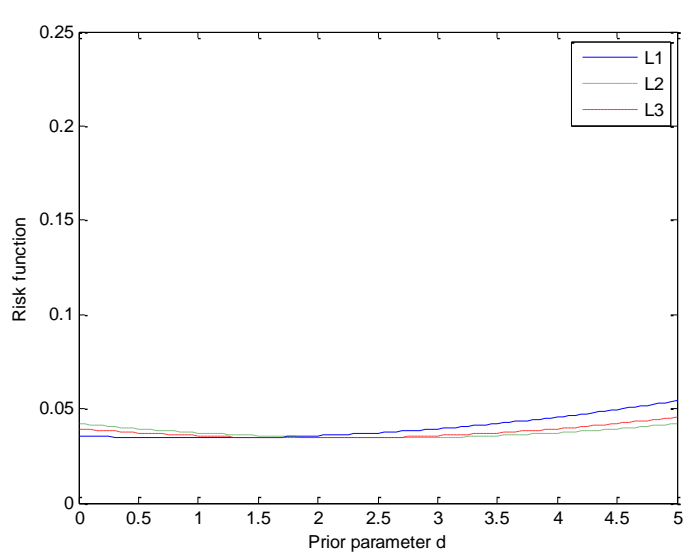

Fig. 3: Ratio of the risk functions with $n=30$

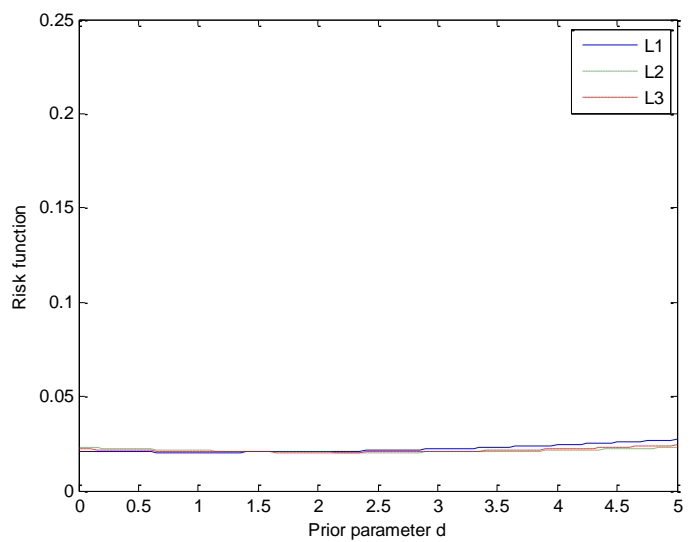

Fig. 4: Ratio of the risk functions with $n=50$

From Fig.1-4, it is clear that no of the estimators uniformly dominates any other. We therefore recommend that the estimators be chosen according to the value of $d$ when the quasi-prior density is used as the prior distribution, and this choice in return depends on the situation at hand. 


\section{References}

[1] Arnold B C , Balakrishnan,N , Nagaraja,H N. Records[M]. John Wiley \&Sons, NewYork, 1998

[2] Raqab, M Z. Inferences for generalized exponential distribution based on records statistics[J]. J.Statist.Plan. Inference, 2002, 104(2): 339-350

[3] Jaheen, Z F.Empirical Bayes analysis of record statistics based on LINEX and quadratic loss functions[J]. Computers and Mathematics with Applications, 2004,47:947-954

[4] Ahmadi , J, Doostparast, $\mathrm{M}$ and Parsian, A. Estimation and prediction in a two parameter exponential distribution based on k-record values under LINEX loss function[J]. Comm. Statist. Theory and Methods, 2005, 34:795-805.

[5] Bain, L J. Statistical Analysis of Reliability and Life Testing Models[M]. Marcer Dekker, New York, 1978.

[6] Chandrasekar, B, Alexander,T L and Balakrishnan, N. Equivariant estimation for parameters of exponential distributions based type-II progressively censored samples[J]. Communications in Statistics. Theory and Methods, 2002, 31(1):1675-1686.

[7] Varian, H R. A Bayesian approach to real estimate assessment[A]. In:Studies in Bayesian Econometrics and statistics in Honor of L.J.Savage, Amsterdam,North Holland, 1975,195-208,.

[8] Zellner, A. Bayesian estimation and prediction using asymmetric loss function[J]. Journal of American statistical Association,1986, 81:446-451.

[9] Podder, C K, Roy, M K, Bhuiyan, K J and Karim, A. Minimax estimation of the parameter of the Pareto distribution for quadratic and MLINEX loss functions[J]. Pak. J. Statist. , 2004, 20(1):137-149.

[10] Kiapoura A, Nematollahib N. Robust Bayesian prediction and estimation under a squared log error loss function[J].Statistics \& Probability Letters,2011, 81(11):1717-1724

[11] Mahmoodi, E, Farsipour,N S.Minimax estimation of the scale parameter in a family of transformed chi-square distributions under asymmetric square $\log$ error and MLINEX loss functions[J]. Journal of sciences, Islamic republic of Islamic, 2006, 17(3):253-258.

[12] Dey,D K , Ghosh, M and Srinivasan, C. Simultaneous estimation of parameters under entropy loss[J],J. Statist. Plan. and Infer.,1987,15:347-363

[13] Singh S K , Singh U , Kumar D.Bayesian estimation of the exponentiated Gamma parameter and reliability function under asymmetric loss function.[J] REVSTAT, 2011, 9(3): 247-260

[14] Li Jin Ping, Ren Hai Ping. Estimation of oneparameter exponential family under entropy loss function based on record values[J]. International Journal of Engineering and Manufacturing, 2012,4: 84-92

\section{Author's Profiles}

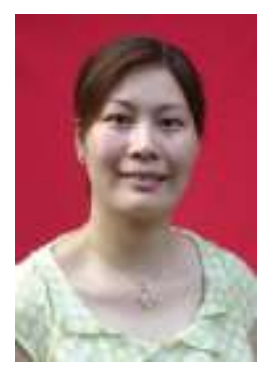

analysis.
Lanping Li: received her B.S. in mathematics from Hunan Normal University in 2002 and she received her M.S. from Hunan Normal University in 2005. She is now a lecture in Hunan University of Finance and Economics. Her research interests are in the areas of Bayes statistics, software reliability

How to cite this paper: Lanping Li,"Minimax Estimation of the Parameter of Exponential Distribution based on Record Values", International Journal of Information Technology and Computer Science(IJITCS), vol.6, no.3, pp.47-53, 2014. DOI: 10.5815/ijitcs.2014.03.06 